\title{
Reconstruction Technique for Defects of the Cutaneous and Mucosal Lip: V-to-flying-Y Closure
}

\author{
Dustin Portela, DO; Stetson Albertson, DO; Jeremy Grekin, MD; Michael Whitworth, DO
}

Repair of a surgical defect following Mohs micrographic surgery can be particularly challenging when it involves free margins of the face. Lip reconstruction falls under this category, requiring careful planning of the repair to avoid not only aesthetic but also functional complications of the oral aperture. Our technique, termed $V$-to-flying-Y closure, allows for an aesthetically pleasing and functional outcome for repairs of surgical defects that involve both the cutaneous and mucosal lip.

Cutis. 2021;107:255-257.

\section{Practice Gap}

Reconstruction of a lip defect poses challenges to the dermatologic surgeon. The lip is a free margin, where excess tension can cause noticeable distortion in facial aesthetics. Distortion of that free margin might not only disrupt the appearance of the lip but affect function by impairing oral competency and mobility; therefore, when choosing a method of reconstruction, the surgeon must take free margin distortion into account. Misalignment of the vermilion border upon reconstruction will cause a poor aesthetic result in the absence of free margin distortion. When a surgical defect involves more than one cosmetic subunit of the lip, great care must be taken to repair each subunit individually to achieve the best cosmetic and functional results.

The suitability of traditional approaches to reconstruction of a defect that crosses the vermilion border-healing by secondary intention, primary linear repair, full-thickness wedge repair, partial-thickness wedge repair, and combined cutaneous and mucosal advancement ${ }^{1}$ — depends on the depth of the lesion.

\section{Clinical Presentation}

A 66-year-old woman with a $4 \times 6-\mathrm{mm}$, invasive, welldifferentiated squamous cell carcinoma of the left lower lip was referred for Mohs micrographic surgery. Removal of the tumor required 2 stages to obtain clear margins, leaving a $1.0 \times 1.2-\mathrm{cm}$ defect that crossed the vermilion border (Figure, A). How would you repair this defect?

\section{Selecting a Technique to Close the Surgical Defect}

For this patient, we had several options to consider in approaching closure, including several that we rejected. Because the defect crossed cosmetic subunit boundaries, healing by secondary intention was avoided, as it would cause contraction, obliterate the vermilion border, and result in poor functional and cosmetic results. We decided against primary closure, even with careful attention to reapproximation of the vermilion border, because the width of the defect would have required a large Burow triangle that extended into the oral cavity. For defects less than one-third the width of the lip, full-thickness wedge repair can yield excellent cosmetic results but, in this case, would decrease the oral aperture and was deemed too extensive a procedure for a relatively shallow defect. ${ }^{2}$

Instead, we chose to perform repair with $\mathrm{V}$ to $\mathrm{Y}$ advancement of skin below the cutaneous defect, up to the location of the absent vermilion border, combined with small, horizontal, linear closure of the mucosal portion of the defect. This approach is a variation of a repair described by Jin et al, ${ }^{3}$ who described using 2 opposing $\mathrm{V}-\mathrm{Y}$ advancement flaps to repair defects of the lip. This 
repair has provided excellent cosmetic results for a small series of our patients, preserving the oral aperture and maintaining the important aesthetic location of the vermilion border. In addition, the technique makes it unnecessary for the patient to undergo a much larger repair, such as a full- or partial-thickness wedge when the initial defect is relatively shallow.

\section{Closure Technique}

It is essential to properly outline the vermilion border of the lip before initiating the repair, ideally before any infiltration of local anesthesia if the surgeon anticipates that tumor extirpation might cross the vermilion border.

Repair-Closing then proceeds as follows:

- The cutaneous portion of the defect is drawn out in standard $V$ to $Y$ fashion, carrying the incision through the dermis and into subcutaneous tissue. The pedicle of the flap is maintained at the base of the island, serving as the blood supply to the flap.

- The periphery of the flap and surrounding tissue is undermined to facilitate movement superiorly into the cutaneous portion of the defect.
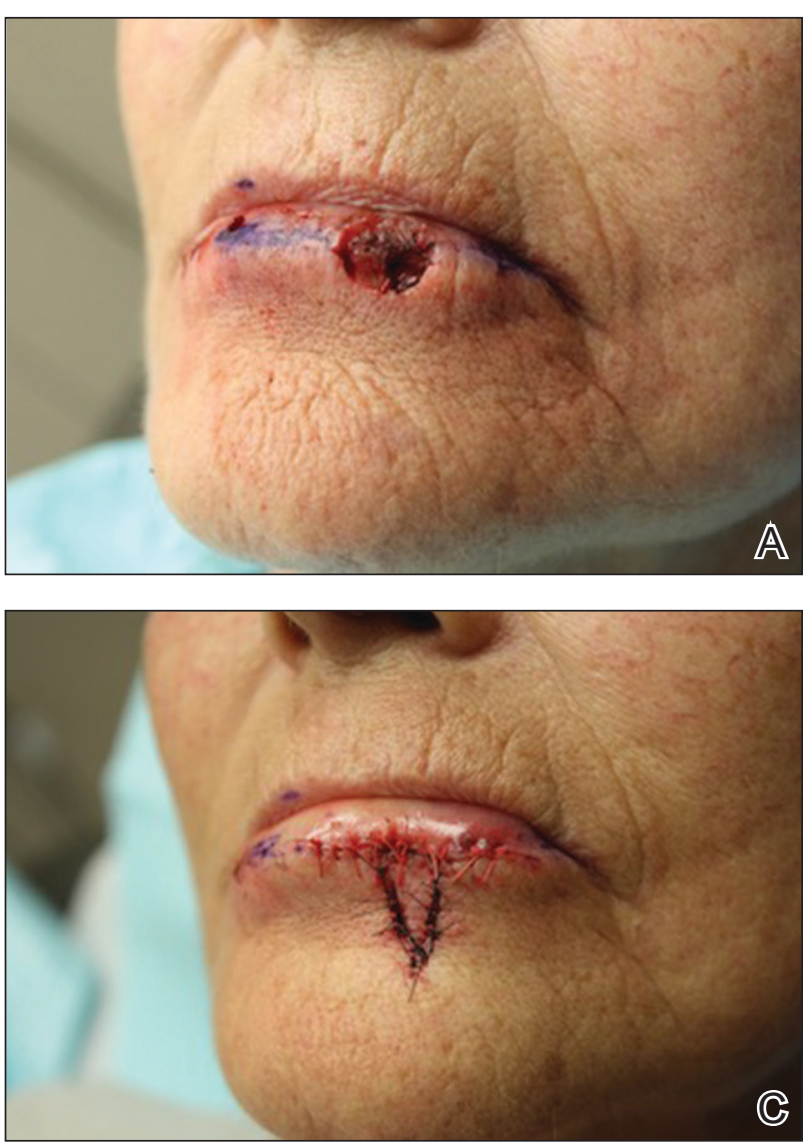

- A single buried vertical mattress suture can be placed at the advancing border of the island, holding it in place at the anticipated location of the vermilion border. The secondary defect created by the advancing $\mathrm{V}$ is closed to help push the island into place and prevent downward tension on the free margin of the lip.

- The remaining defect of the vermilion lip is closed by removing Burow triangles at the horizontal edges on each side of the remaining defect (Figure, B). The triangles are removed completely within the mucosal lip, with the inferior edge of the triangle placed at the vermilion border.

- The defect is closed in a primary linear horizontal fashion, using buried vertical mattress sutures and cutaneous approximation.

The final appearance of the sutured defect yields a small lateral extension at the superior edge of the $\mathrm{V}$ to $Y$ closure, giving the appearance of wings on the $Y$, prompting us to term the closure $V$-to-flying- $Y$ (Figure, C).

Although the limited portion of the mucosal lip that is closed in this fashion might appear thinner than the remaining lip, it generally yields a cosmetically acceptable
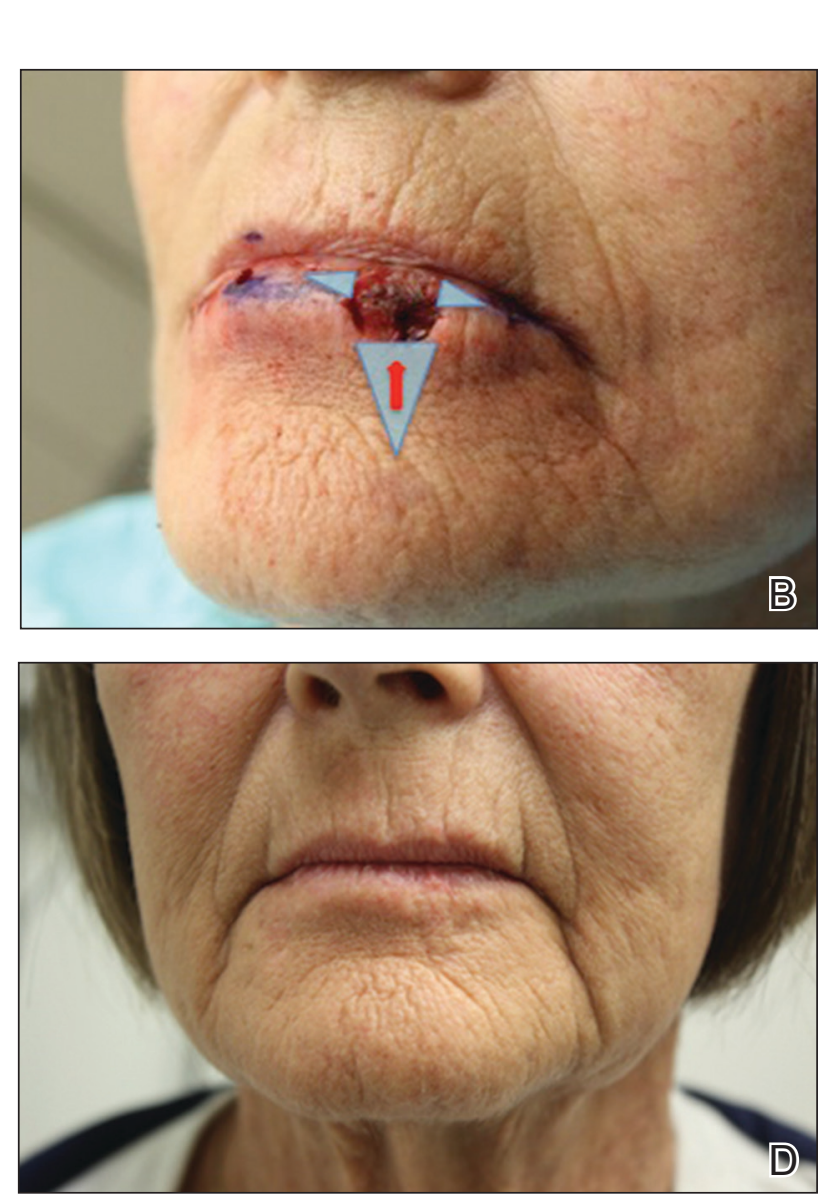

A, A $1 \times 1.2-c m$ defect of the lower lip, involving the vermilion and cutaneous lip. The vermilion border is outlined prior to the start of extirpation of the tumor. B, Illustration of the proposed incision lines and direction of tissue movement (arrow). C, Completed repair, demonstrating proper placement of the vermilion border and lack of displacement of the lip. D, At 11-month follow-up, there is no distortion of the vermilion border. The patient reports no functional deficit. 
result in the properly selected patient. Our experience also has shown an improvement in this difference in the months following repair. A full mucosal advancement flap might result in a more uniform appearance of the lower lip, but it is a larger and more difficult procedure for the patient to endure. Additionally, a full mucosal advancement flap risks uniformly creating a much thinner lip.

Postoperative Course-Sutures were removed 1 week postoperatively. Proper location of the vermilion border, without distortion of the free margin, was demonstrated. At 11-month follow-up, excellent cosmetic and functional results were noted (Figure, D).

\section{Practice Implications}

This repair (1) demonstrates an elegant method of closing a relatively shallow defect that crosses the vermilion border and (2) allows the surgeon to address each cosmetic subunit individually. We have found that this repair provides excellent cosmetic and functional results, with little morbidity.

The lip is a common site of nonmelanoma squamous cell carcinoma. Poorly planned closing after excision of the tumor risks notable impairment of function or cosmetic distortion. When a defect of the lip crosses cosmetic subunits, it is helpful to repair each subunit individually. V-to-flying-Y closure is an effective method to close defects that cross the vermilion border, resulting in wellpreserved cosmetic appearance and function.

\section{REFERENCES}

1. Ishii LE, Byrne PJ. Lip reconstruction. Facial Plast Surg Clin North Am. 2009;17:445-453. doi:10.1016/j.fsc.2009.05.007

2. Sebben JE. Wedge resection of the lip: minimizing problems. J Dermatol Surg Oncol. 1985;11:60-64. doi:10.1111/j.1524-4725.1985.tb02892.x

3. Jin X, Teng L, Zhang C, et al. Reconstruction of partial-thickness vermilion defects with a mucosal V-Y advancement flap based on the orbicularis oris muscle. J Plast Reconstr Aesthet Surg. 2011;64:472-476. doi:10.1016/j.bjps.2010.07.017 